\title{
Evidence of columnar order in the fully frustrated transverse field Ising model on the square lattice
}

\author{
Sandro Wenzel, ${ }^{1}$ Tommaso Coletta, ${ }^{1}$ Sergey E. Korshunov, ${ }^{2}$ and Frédéric Mila ${ }^{1, *}$ \\ ${ }^{1}$ Institute of Theoretical Physics, École Polytechnique Fédérale de Lausanne (EPFL), CH-1015 Lausanne, Switzerland \\ ${ }^{2}$ L. D. Landau Institute for Theoretical Physics RAS, 142432 Chernogolovka, Russia
}

(Dated: August 30, 2018)

\begin{abstract}
Using extensive classical and quantum Monte Carlo simulations, we investigate the ground-state phase diagram of the fully frustrated transverse field Ising model on the square lattice. We show that pure columnar order develops in the low-field phase above a surprisingly large length scale, below which an effective $U(1)$ symmetry is present. The same conclusion applies to the Quantum Dimer Model with purely kinetic energy, to which the model reduces in the zero-field limit, as well as to the stacked classical version of the model. By contrast, the $2 \mathrm{D}$ classical version of the model is shown to develop plaquette order. Semiclassical arguments show that the transition from plaquette to columnar order is a consequence of quantum fluctuations.
\end{abstract}

The Ising model in a transverse field, introduced by De Gennes in the context of ferroelectric systems [1] and defined by the Hamiltonian

$$
H_{\mathrm{TFIM}}=\sum_{\langle i j\rangle} J_{i j} \sigma_{i}^{z} \sigma_{k}^{z}-\Gamma \sum_{j} \sigma_{j}^{x}
$$

where $\sigma^{\alpha}$ are Pauli matrices, $\Gamma$ the transverse field and $J_{i j}$ Ising coupling constants, has imposed itself as one of the most useful minimal models to study quantum fluctuations [2]. In particular, the simple ferromagnetic version of the model undergoes, when the transverse field is increased, a phase transition to a paramagnetic phase that embodies several of the basic aspects of quantum phase transitions [3]. Frustrated versions of the model, i.e. versions in which it is impossible to minimize the energy for all bonds simultaneously, have been studied over the years [4], with in general very rich phase diagrams [2].

Recently, special attention has been payed to the subclass of Fully Frustrated Transverse Field Ising Models (FFTFIM) $[5,6]$. In these models, the couplings are limited to nearest neighbors and have equal magnitudes, but their signs are such that it is impossible to satisfy simultaneously all bonds around any elementary plaquette, resulting in a very strong ground state degeneracy. The AF Ising model on the triangular lattice is a typical example. On the square lattice, this can be achieved if the number of antiferromagnetic couplings around any elementary plaquette is odd, resulting into an Ising model often referred to as Villain's odd model [7]. All Ising models satisfying this condition are equivalent, being related by a local gauge transformation. In the present paper, we use the gauge with antiferromagnetic (positive) couplings $\left(J_{i j}=J\right)$ on every second vertical line, and ferromagnetic (negative) couplings $\left(J_{i j}=-J\right)$ for all other bonds.

The interest in this family of models has raised because of their connection to the Quantum Dimer Model (QDM) of Rokhsar and Kivelson [8]. First introduced in the context of the Resonating Valence Bond (RVB) theory of high $\mathrm{T}_{c}$ cuprate superconductors, the QDM has become one of the paradigmatic models in the field of quantum spin liquids after Moessner and Sondhi have shown that, on the triangular lattice, it has an RVB phase [9]. On the square lattice, the QDM is defined by

$$
\begin{aligned}
& H_{\mathrm{QDM}}=\sum[-t(\mid \because ⿱ \therefore)\langle\mathfrak{!}|+\text { h.c. })+ \\
& V(|\because \because\rangle\langle\because[+|\mathfrak{!}\rangle\langle\mathfrak{!} !)],
\end{aligned}
$$

(in terms of hard core dimer objects $! !$ ) where $t$ and $V$ are the amplitudes of kinetic and potential terms, and the sum runs over all elementary square plaquettes. In the limit $\Gamma \rightarrow 0$, and up to a trivial degeneracy associated to a global spin flip, the FFTFIM maps onto the purely kinetic QDM with $V / t=0$ $[5,6]$.

Over the last few years, the properties of the QDM on several lattices have been investigated in great details, and in most cases a consensus has been reached regarding its phase diagram as a function of $V / t$ [10], with one noticeable exception: on the square lattice, while it is clear that the QDM has a columnar phase for $V \rightarrow-\infty$ and a staggered phase for $V / t>1$, the situation between these two limits is still controversial. It was suggested on the basis of exact diagonalizations (ED) that the system has columnar order up to $V / t=1$ [11], but subsequent investigations have challenged this picture. ED on larger systems have been interpreted as evidence of plaquette order in the range $(V / t)_{c}<V / t<1$ with $(V / t)_{c} \simeq-0.2$ [12]. The same conclusion has been reached with Quantum Monte Carlo (QMC), with a critical ratio $(V / t)_{c} \simeq 0.6$ significantly closer to 1 though [13]. Finally, a new scenario [14] has been put forward on the basis of ED, Green's function QMC, and a mapping onto a height model [15], according to which a mixed phase is present between $V / t \simeq 0$ and $V / t \simeq 0.8$, in which all symmetries broken in the columnar and plaquette phases are simultaneously broken. This scenario has been supported by QMC on a related hard-core boson model [16] and is not in direct conflict with Ref. [13] whose analysis was not designed to be sensitive to the presence of a mixed phase.

A major problem in studying the competition between columnar and plaquette order in the QDM on the square lattice is that there is no simple limit where the plaquette phase is known to exist for sure. In this Letter, we investigate the same competition in the context of the FFTFIM. The main advantage is that, as we shall see, the FFTFIM has a plaquette phase in a simple limit, that of classical spins. A combination 


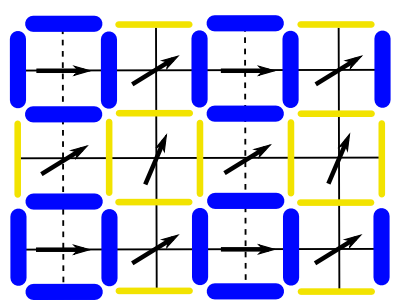

(a)

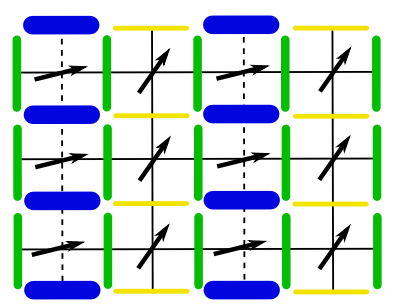

(b)

FIG. 1. (color online). Visualization of the square lattice structure (black solid lines for ferromagnetic couplings and dashed lines for AF couplings) and of particular classical spin configurations with corresponding dimer configurations on the dual lattice: (a) plaquette dimer structure and (b) columnar dimer structure. Dimer densities on the dual lattice (encoded by the thickness of color lines) correspond to bond energies on the original spin lattice as described in the text.

of large scale QMC simulations and semiclassical arguments shows that the plaquette phase disappears when quantum fluctuations are introduced, and that pure columnar order is stabilized from the paramagnetic phase down to zero transverse field, hence also for the QDM for $V / t=0$, with no indication of a mixed phase.

A convenient gauge-invariant description of ordering in the FFTFIM can be introduced [17, 18] by using the energies of bonds $\left\langle i i+\mathbf{e}_{\alpha}\right\rangle$ of the original lattice to define dimer densities $d_{i, \alpha}=\frac{1}{2}\left(1-J_{i i+\mathbf{e}_{\alpha}} / J \sigma_{i}^{z} \sigma_{i+\mathbf{e}_{\alpha}}^{z}\right)$ on the bonds of the dual lattice crossing them. This mapping gives rise to the visualization of two typical dimer-solid states shown in Fig. 1. It allows one to characterize all aforementioned dimer-solid phases by a single complex order parameter $\Psi[11,12,16,19,20]$, which in terms of $\tilde{d}_{\alpha}(\mathbf{q})=\frac{1}{N} \sum_{i} e^{\mathrm{i} \mathbf{q} \cdot \mathbf{r}_{i, \alpha}} d_{i, \alpha}$, the Fourier transforms of dimer density $d_{i, \alpha}$, has a very simple form,

$$
\Psi=2\left[\tilde{d}_{x}(0, \pi)+\mathrm{i} \tilde{d}_{y}(\pi, 0)\right] .
$$

Long-range ordering into some sort of valence bond solid is indicated by $\langle|\Psi|\rangle>0$, while the precise structure of the solid is controlled by the phase $\phi$ of the order parameter $\Psi=|\Psi| e^{\mathrm{i} \phi}$ [16]: The columnar phase is characterized by a purely real or imaginary $\Psi$, i.e., $\phi=\phi_{c}=n \pi / 2(n=0, \ldots, 3)$, the plaquette state by a phase angle $\phi=\phi_{p}=\pi / 4+n \pi / 2(n=0, \ldots, 3)$, and the mixed-state by a phase $\phi$ which is neither of the form $\phi_{c}$ nor $\phi_{p}$. Instead of directly studying the phase $\phi$, we find it more convenient to look at a phase sensitive order parameter $[21,22]$ defined as

$$
\Psi_{4}=\langle|\Psi| \cos (4 \phi)\rangle
$$

It is straightforward to see that in a columnar phase $\Psi_{4} \rightarrow|\Psi|$ whereas $\Psi_{4} \rightarrow-|\Psi|$ holds for plaquette ordering. In a true mixed phase, however, $\left|\Psi_{4}\right|$ will not converge towards $|\Psi|$ in the thermodynamic limit.

In addition, other studies $[14,16]$ used special order parameters $P_{+}$and $P_{-}$, based on plaquette structure factors [23], to measure plaquette and columnar ordering correlations separately. We will show, however, that in the present context those have to be analyzed with care.

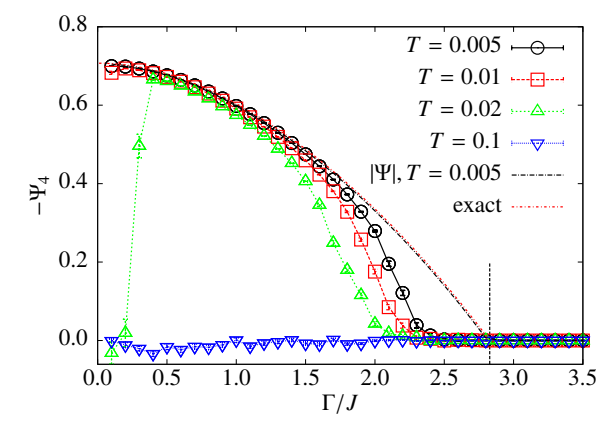

FIG. 2. (color online). Plot of the order parameter $-\Psi_{4}$ for the classical limit of (1) at linear lattice size $L=16$ and various low temperatures $T$ confirming the nature of the ground state to be a plaquette state. For comparison, the norm $|\Psi|$ is shown at $T=0.005$ as well as calculated from the exactly known ground state. The exact zerotemperature critical field $\Gamma_{c} / J=2 \sqrt{2}$ is indicated by the vertical line.

Classical limit. - The classical limit of Eq. (1) defined by interpreting $\sigma^{z}$ and $\sigma^{x}$ as components of an ordinary threedimensional vector of length $S$ has previously been used to study the FFTFIM on the honeycomb lattice [18], which can be mapped onto the QDM on the triangular lattice in the limit of weak field, and its predictions, when combined with linear spin-wave theory (LSWT), agree very well with QMC results on the QDM. For the FFTFIM on the square lattice, the paramagnetic phase with spins along the $x$ direction can be shown to extend down to $\Gamma_{c} / J=2 \sqrt{2}$, and in the range $0<\Gamma / J<2 \sqrt{2}$, a single phase is stabilized with a four-site unit cell and one spin per unit cell fully polarized along the field. This has been checked numerically and can be proven analytically in the limits $\Gamma \rightarrow 0$ and $\Gamma \rightarrow \Gamma_{c}^{-}$[24]. This phase corresponds to a plaquette state in the dimer language (see Fig. 1(a)). The columnar state would correspond to the dimer density pattern sketched in Fig. 1(b). Minimizing the energy for a unit cell which has the symmetry of the columnar state leads to a configuration which is a saddle point of the total energy, but not a local minimum, and a fortiori not a global one.

This result is quite surprising in view of the most recent results obtained for the QDM at $V / t=0$, which suggest a columnar or a mixed phase, but not a plaquette phase. In order to confirm it and to test the order parameter $\Psi$ introduced above, we have performed finite temperature MC simulations of the classical version of (1) on systems of size $L$ with periodic boundary conditions. They fully corroborate our analysis, see Fig. 2. In particular, we find a convergence of the phase sensitive quantity $-\Psi_{4} \rightarrow|\Psi|$ in the ordered region $\Gamma<\Gamma_{c}$, proving the presence of the plaquette state in the classical FFTFIM.

The stacked Ising model.- Motivated by this somehow unexpected result, and as a first step towards a full quantum treatment of the FFTFIM, we have decided to use the same type of MC simulations to revisit the stacked fully frustrated Ising model first studied by Jalabert and Sachdev [19]. This 

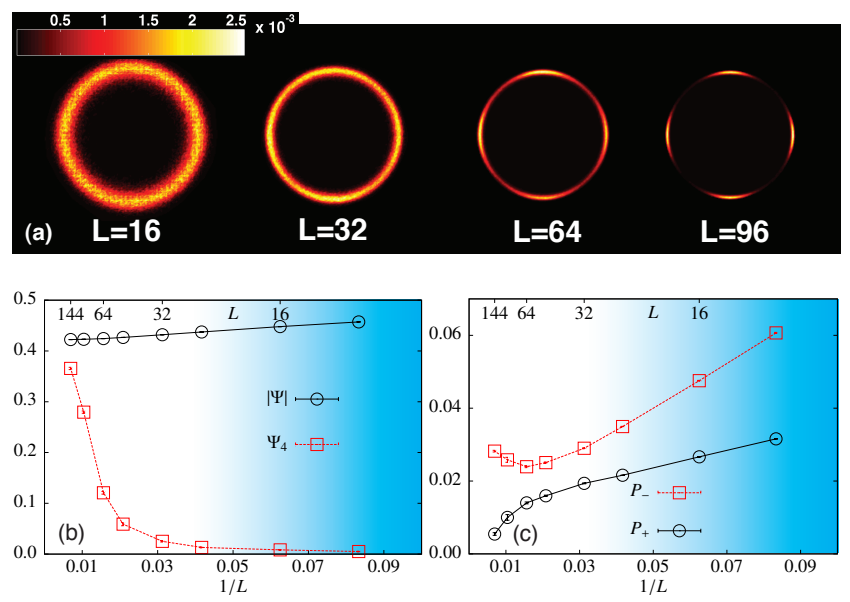

FIG. 3. (color online). (a) Order parameter distribution function (in the complex $\Psi$-plane) for the stacked magnet at $T=1.5$ for various lattice sizes. (b) Finite-size behavior of the two order parameters $\Psi$ and $\Psi_{4}$ versus $1 / L$. (c) Same as (b) for the order parameters $P_{+}$ (plaquette correlations) and $P_{-}$(columnar correlations). The color shaded regions indicate the approximate range where almost perfect $U(1)$-symmetric distributions are found. All results demonstrate the presence of columnar dimer solid in the stacked magnet.

model is closely related to the FFTFIM since they can be mapped onto each other using Trotter-Suzuki's decomposition if appropriately scaled coupling constants and stack dimension are used, and Jalabert and Sachdev have concluded that columnar order is stabilized in the stacked model when the temperature $T$ is finite. However, what they actually showed is that there is a phase transition at $T \simeq 2.85$ below which $|\Psi|$ acquires a finite value without actually resolving the phase of the order parameter. We have performed high-statistics MC simulations inside the ordered phase at $T=1.5$, with a focus on obtaining such conclusive phase-sensitive information. The resulting distribution functions of the complex order parameter $\Psi$ for linear lattice sizes $L=16,32,64,96$ are shown in Fig. 3(a). Interestingly, a $U(1)$ symmetric distribution is found for $L \lesssim 32$, but ultimately four peaks develop at phase angles $\phi=\phi_{c}$ and a clear columnar state emerges above the $U(1)$ regime. These results lead to two important conclusions: (i) Quantum fluctuations seem to change the nature of ordering from plaquette to columnar; (ii) A $U(1)$ length scale (as in [22]) prohibits to draw any conclusion from data on small system sizes.

It is instructive to study the effect of this length scale on the finite-size scaling of order parameters. Figure $3(b, c)$ compares the finite-size dependence of $|\Psi|$ and $\Psi_{4}$. It is only for $L \gtrsim 32$ that a clear signal appears showing that $\Psi_{4}$ will eventually converge towards $|\Psi|$. For the order parameters $P_{+}$and $P_{-}$, the effect is even more dramatic (see Fig. 3(c)): The results for sizes up to $L=40$ are clearly consistent with a finite value for both $P_{+}$and $P_{-}$in the thermodynamic limit, hence with a mixed state, as in the analysis of Refs. $[14,16]$, but larger sizes reveal that $P_{+}$finally drops to zero while $P_{-}$starts increasing again to reach a much larger value than expected, a
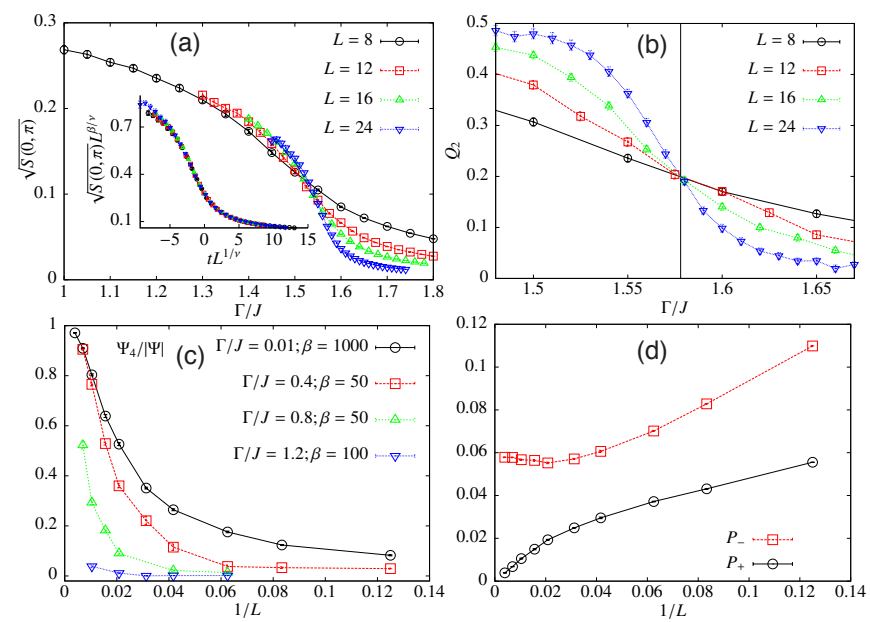

FIG. 4. (color online). (a) Spin structure factor $S(0, \pi)$ as a function of the transverse field $\Gamma$ indicating an ordered phase below $\Gamma / J \lesssim$ 1.6 (Inset : Scaling of $S(0, \pi)$ with the critical exponents from the $X Y$ universality class.) (b) Associated Binder parameter. It gives a precise estimate of $\Gamma_{c} / J$ indicated by the vertical line. (c) Scaling of the order parameter ratio $\Psi_{4} / \Psi$ for the quantum FFTFIM for various values of the field $\Gamma$. It provides convincing evidence of columnar ordering for the smallest fields. (d) Analysis of the order parameters $P_{+}$and $P_{-}$for the smallest field studied $\Gamma / J=0.01$.

clear indication of a pure columnar state.

The quantum FFTFIM. - Next, we turn to a direct analysis of the ground-state phase diagram of Eq. (1) for arbitrary field $\Gamma$. Using continuous time and sign-problem free quantum Monte Carlo methods $[25,26]$ on periodic lattices with linear system sizes up to $L=256$, we investigate the order parameter $\Psi$ and the basic ordering modes in terms of the (sublattice) structure factors $S(0,0)$ and $S(0, \pi)$ (see, Fig. 1(b)). In each case, the inverse temperature $\beta$ was carefully chosen to be large enough to sample ground-state properties. Results for the spin structure factors are reproduced in Fig. 4(a), which show that an ordered phase is stabilized by quantum fluctuations below $\Gamma / J \lesssim 1$.6. A more precise estimate of the critical transverse field is obtained from the Binder parameter $Q_{2}=1-\left\langle S(0, \pi)^{2}\right\rangle /\left(3\langle S(0, \pi)\rangle^{2}\right)$ which is scale invariant at the quantum critical point. The crossings of $Q_{2}$ lead to the estimate $\Gamma_{c} / J=1.578(2)$, see Fig. 4(b). This critical field is considerably reduced as compared to the classical value $2 \sqrt{2}$. Using the value obtained for $\Gamma_{c}$ and $3 \mathrm{D} X Y$ critical exponents $(\beta$ and $v$ ) [27], a good data collapse of the structure factors is obtained, directly demonstrating the 3D $X Y$ universality class of the quantum critical point. This result coherently adds to the indirect analysis performed in Ref. [21] and supports the field-theoretic picture $[28,29]$ beyond reasonable doubt. It also confirms the similarity between the triangular and square lattice FFTFIM as far as the critical behavior is concerned. An analysis of the FFTFIM at finite temperature that reveals the presence of an extended critical phase will be presented elsewhere [24]. To determine the nature of the phase(s) for $\Gamma<\Gamma_{c}$, we calculate the order parameter $\Psi_{4}$ and analyze its 
finite-size scaling with respect to $|\Psi|$. Figure 4(c) shows the essential results of this analysis for a couple of selected field values inside the ordered region. While it is clearly difficult to get phase sensitive information close to the quantum critical point (for $\Gamma / J \simeq 1.2$ and above) - continuous distributions as in Fig. 3 (top) are seen up to the largest system size - we note that it actually becomes easier to obtain structural information in the limit $\Gamma / J \rightarrow 0$. Indeed, for $\Gamma / J=0.01$ the scaling of $\Psi_{4}$ is sufficient to conclude on the presence of a columnar state, a result fully supported by the behavior of $P_{+}$and $P_{-}$, see Fig. 4(d). Based on this analysis, we can safely conclude that the columnar state is stabilized at least up to $\Gamma / J \approx 1.0$. Above this value, it is currently difficult to analyse the nature of the phase by means of finite-lattice simulations. This particular difficulty close to the quantum critical point is actually not surprising since the 3D XY quantum critical point has a $U(1)$ symmetry which is at the origin of the disturbing crossover behavior found here [22]. A similar observation has already been made in the case of the triangular lattice [21].

Semiclassical approach. - All these results indicate that the order in the low field phase changes from plaquette to columnar upon going from the classical to the ultra-quantum spin-1/2 case. In order to investigate this issue from a complementary angle, we have studied how quantum fluctuations may favor columnar order in the context of a semiclassical approach. As stated earlier, the classical solution which corresponds to columnar order is not a local minimum of the energy. So the spectrum of the harmonic fluctuations cannot be diagonalized by a Bogoliubov transformation. An upper bound of the zero point energy can nevertheless be obtained by applying local fields in the direction of the spins to achieve the positiveness of the quadratic form that can be diagonalized by a Bogoliubov transformation (see Supplemental Material). For the plaquette state, the Hamiltonian of the quadratic fluctuations can be diagonalized by a Bogoliubov transformation. The phase diagram obtained by comparing the energy of the columnar and plaquette states corrected in this way by quantum fluctuations is shown in Fig. 5. The results nicely confirm the anticipated transition: upon reducing the spin, quantum fluctuations reduce the energy of the columnar state much faster than that of the plaquette one and stabilize it for all fields for spin-1/2.

Conclusions. - From the various results reported in this paper, a number of firmly established conclusions can be drawn. First of all, at low field, the ground state of the quantum FFTFIM on the square lattice is an ordered phase whose structure corresponds, in the gauge invariant dimer language, to columnar order. This order is stable at least up to $\Gamma / J=1$, and possibly up to the transition to the paramagnetic phase, which has been shown to take place at $\Gamma_{c} / J \simeq 1.58$. By contrast, the classical version of the model develops an order with a four-spin unit cell at low temperature which, in the dimer language, corresponds to plaquette order. Furthermore, the robustness of plaquette order with respect to two types of perturbations, stacking and quantum fluctuations, has been tested, with the conclusion that it is relatively fragile: 3D stacking

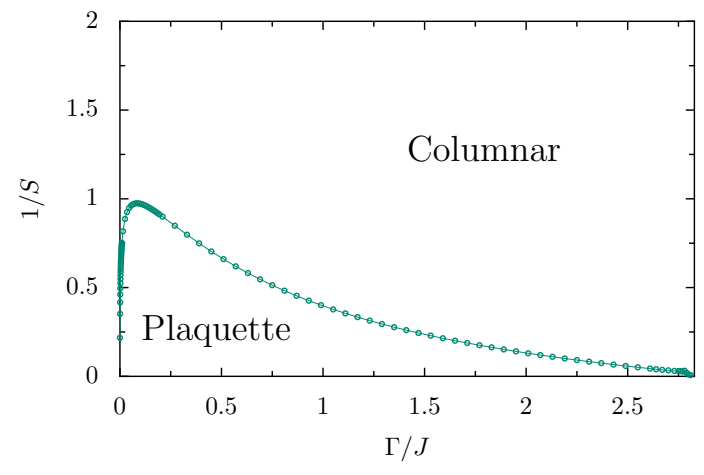

FIG. 5. (color online). Phase diagram in the $\Gamma / J-1 / S$ plane, showing the phase boundary between columnar and plaquette phase where the transition line shown is an upper bound for the region of the stability of the plaquette phase. The columnar phase is clearly stabilized by quantum fluctuations.

clearly stabilizes columnar order, while quantum fluctuations treated within linear spin-wave theory stabilize columnar order in a large portion of the $(1 / S, \Gamma / J)$ phase diagram, and in particular for all values of $\Gamma / J$ outside the paramagnetic phase for the quantum model with $S=1 / 2$.

Regarding the closely related QDM on the square lattice, the present results clearly demonstrate that, in the purely kinetic regime $V / t=0$, which is equivalent to the $\Gamma / J \rightarrow 0$ limit of the FFTFIM, columnar order is stabilized. In addition, this is likely to be the case in a finite parameter range around this point since we have not seen any particular indication of a proximity to a critical point. Complementary work carried out in parallel to ours comes to similar conclusions [30].

Finally, the other important message of this paper is the presence of a surprisingly large length scale below which the model has an effective U(1) symmetry. This makes the identification of the actual order stabilized in the thermodynamic limit particularly difficult and, to a large extent, explains the conflicting results obtained so far on the type of order stabilized in the QDM between $V / t=0$ and $V / t=1$. By studying very large systems, we have been able to beat this length scale up to $\Gamma / J \simeq 1$. Beyond that value, the proximity of the transition to the paramagnetic phase makes this length scale even larger, and whether columnar order remains stable up to the paramagnetic phase, as suggested by the semiclassical approximation, could not be numerically decided.

We thank P. Corboz, S. Isakov, A. Läuchli, and S. Wessel for fruitful discussions and C. Henley for comments on the manuscript. This work was supported by the Swiss National Fund and by MaNEP.

* frederic.mila@epfl.ch

[1] P. de Gennes, Solid State Communications 1, 132 (1963).

[2] B. K. Chakrabarti, A. Dutta, and P. Sen, Quantum Ising Phases and Transitions in Transverse Ising Models (Springer Verlag, 
Heidelberg, 1996).

[3] S. Sachdev, Quantum Phase Transitions (Cambridge University Press, 2001).

[4] R. Liebmann, ed., Statistical Mechanics of Periodic Frustrated Ising Systems, Lecture Notes in Physics, Vol. 251 (Springer Verlag, Berlin, 1986).

[5] R. Moessner, S. L. Sondhi, and P. Chandra, Phys. Rev. Lett. 84, 4457 (2000).

[6] R. Moessner and S. L. Sondhi, Phys. Rev. B 63, 224401 (2001).

[7] J. Villain, J. Phys. C: Solid State Physics 10 (1977).

[8] D. S. Rokhsar and S. A. Kivelson, Phys. Rev. Lett. 61, 2376 (1988).

[9] R. Moessner and S. L. Sondhi, Phys. Rev. Lett. 86, 1881 (2001).

[10] For a recent review see, e.g., R. Moessner and K. S. Raman, "Introduction to frustrated magnetism: Materials, experiments, theory," (Springer, 2011) Chap. Quantum dimer models, pp. 437-479.

[11] S. Sachdev, Phys. Rev. B 40, 5204 (1989).

[12] P. W. Leung, K. C. Chiu, and K. J. Runge, Phys. Rev. B 54, 12938 (1996).

[13] O. F. Syljuåsen, Phys. Rev. B 73, 245105 (2006).

[14] A. Ralko, D. Poilblanc, and R. Moessner, Phys. Rev. Lett. 100, 037201 (2008).

[15] C. L. Henley, J. Stat. Phys 89, 483 (1997).

[16] S. Wessel, Phys. Rev. B 78, 075112 (2008).

[17] G. Misguich and F. Mila, Phys. Rev. B 77, 134421 (2008).

[18] T. Coletta, J.-D. Picon, S. E. Korshunov, and F. Mila, Phys. Rev. B 83, 054402 (2011).

[19] R. A. Jalabert and S. Sachdev, Phys. Rev. B 44, 686 (1991).

[20] A. Sen, K. Damle, and T. Senthil, Phys. Rev. B 76, 235107 (2007).

[21] S. V. Isakov and R. Moessner, Phys. Rev. B 68, 104409 (2003).

[22] J. Lou, A. W. Sandvik, and L. Balents, Phys. Rev. Lett. 99, 207203 (2007).

[23] For each plaquette on the dual lattice, identified by a lattice site $i$ and position $\mathbf{r}_{i}$ on the original lattice, one considers symmetric/antisymmetric operators $k_{ \pm, i}=d_{i, x} d_{i-\mathbf{e}_{x}, x} \pm$ $d_{i, y} d_{i-\mathbf{e}_{y}, y}$ and calculates the equal-time structure factor $S_{ \pm}(\mathbf{q})=$ $\left(1 / N^{2}\right) \sum_{i, j} e^{\mathrm{i} \mathbf{q}\left(\mathbf{r}_{\mathrm{i}}-\mathbf{r}_{\mathrm{j}}\right)} k_{ \pm, i} k_{ \pm, j}$. Then $P_{-}=S_{-}(0,0)^{1 / 2}$ detects columnar correlations and $P_{+}=S_{+}(\pi, \pi)^{1 / 2}$ plaquette correlations.

[24] S. Wenzel, T. Coletta, S. E. Korshunov, and F. Mila, unpublished.

[25] H. Rieger and N. Kawashima, European Physical Journal B 9 , 233 (1999).

[26] T. Nakamura, Phys. Rev. Lett. 101, 210602 (2008).

[27] M. Campostrini, M. Hasenbusch, A. Pelissetto, and E. Vicari, Phys. Rev. B 74, 144506 (2006).

[28] D. Blankschtein, M. Ma, A. N. Berker, G. S. Grest, and C. M. Soukoulis, Phys. Rev. B 29, 5250 (1984).

[29] D. Blankschtein, M. Ma, and A. N. Berker, Phys. Rev. B 30, 1362 (1984).

[30] D. Schwandt, S. Isakov, S. Capponi, R. Moessner, and A. Läuchli, unpublished.

\section{Supplemental Material}

Here we present the details of the analysis of the effect of quantum fluctuations for the plaquette and columnar states. Quantum fluctuations are investigated in the context of a large $S$ expansion (spin wave expansion). In this framework, the fluctuations around a classical solution are described in terms of Holstein-Primakoff bosons and the fluctuation Hamiltonian is truncated at the harmonic level (linear spin wave expansion).

In the two classical structures that are compared, the fluctuation Hamiltonians do not contain terms which are linear in the Holstein-Primakoff bosons and therefore are purely quadratic. In the case of the plaquette state, the correction to the classical energy is obtained by diagonalizing the fluctuation Hamiltonian. This is done in Fourier space via a standard Bogoliubov transformation which yields the dispersion relations and the correction to the classical energy.

The computation in the case of the columnar state is more involved. This time the starting classical configuration is not a minimum but a saddle point of the classical energy. This results in a quadratic fluctuation Hamiltonian which is not positive definite. Trying to diagonalize the quadratic Hamiltonian via a Bogoliubov transformation in this case yields a spectrum which is not well defined for all values of momenta. For the regions in the Brillouin zone near $\vec{k}=(0, \pm \pi)$ the spectrum is not real but takes complex nonphysical values. In this case, the correction to the classical energy cannot be computed. The fact that the spectrum is not well defined results from the truncation of the spin wave approximation to harmonic level. If this state is to become the ground state when quantum fluctuations are included, higher order terms in the spin wave expansion must yield a spectrum with real frequencies. However this implies treating higher order terms self consistently.

In order to have a well-defined spectrum we proceed in a different way. We add to the Hamiltonian a local field term on each site of the form

$$
V=\frac{\delta}{S} \sum_{j}\left(S-\hat{S}_{j}^{z^{\prime}(j)}\right)
$$

where $\delta>0$ parametrizes the local field strength and where the summation is taken over all sites and at each site the axis $z^{\prime}(j)$ is directed along the direction of the classical spin. The field $\delta$ is adjusted to have a fluctuation Hamiltonian which is positive definite allowing it to be diagonalized by a Bogoliubov transformation. The resulting spectrum has real and positive frequencies with soft modes only at the wave vectors $\vec{k}=(0, \pm \pi)$. The advantages of this approach are the following: the addition of $V$ to the Hamiltonian does not change the classical energy of the state considered and allows to obtain dispersion relations which are physically meaningful. Furthermore $V$ is a strictly positive contribution to the Hamiltonian. Hence the corrections to the energy of the columnar state computed with this approach provide an upper bound of the energy of this state at order $1 / S$.

We find that as a function of $S$ and $\Gamma / J$, the upper bound of the energy of the columnar state is lower than the energy of the plaquette state in a significant parameter range (see Fig. 5 of main text). In particular, the columnar state wins for all fields for $S=1 / 2$, the value of the spin for which the model can be exactly mapped onto a QDM with $V / t=0$ in the limit $\Gamma / J \rightarrow 0$. 